\title{
Extraordinary absorption by a thin dielectric slab backed with a metasurface
}

\author{
Ana Díaz-Rubio, Daniel Torrent, ${ }^{*}$ Jorge Carbonell, and José Sánchez-Dehesa ${ }^{\dagger}$ \\ Wave Phenomena Group, Department of Electronics Engineering, Universitat Politècnica de València, Camino de vera s.n. \\ (Edificio 7F1), ES-46022 Valencia, Spain
}

(Received 11 March 2014; revised manuscript received 14 May 2014; published 18 June 2014)

\begin{abstract}
The absorption of electromagnetic waves by a thin dielectric slab backed by a metasurface has been comprehensively studied and discussed at microwave frequencies. The metasurface consists of a metallic plate decorated with a periodic distribution of coaxial- or annular-type cavities. Analytical expressions for the absorbance have been obtained by using a mode-matching method. For $P$-polarized waves it is predicted that a low-frequency peak of perfect absorption is possible by properly choosing the lossy component of the permittivity of the dielectric slab, with the position of this peak being easily tunable by the cavity length. It is also shown that non-Bravais lattices, containing several cavities in the unit cell, and the excitation of guided waves by the periodic array of resonators are complementary absorption mechanisms in the studied structures.
\end{abstract}

DOI: 10.1103/PhysRevB.89.245123

PACS number(s): 78.68.+m, 41.20.-q, 78.20.Ci

\section{INTRODUCTION}

At the beginning of the last century Rayleigh pointed out that flat rigid surfaces with cylindrical holes present acoustic resonances for well-defined hole lengths [1]. Rayleigh also showed that for the resonant conditions the acoustic energy is concentrated in the holes and predicted that a similar effect should occur with electromagnetic (EM) waves. The existence of localized waveguide resonances, equivalent to the acoustic resonances described by Rayleigh, has been extensively demonstrated in metallic gratings [2-4]. For example, in one-dimensional (1D) gratings with deep rectangular cross sections, surface-shape resonances localized in the channels can be excited when the impinging light has an electric-field component perpendicular to the groove direction $[3,4]$.

Metallic gratings can also exhibit absorption anomalies [5] indicative of surface plasmon polariton (SPP) excitations at the air-metallic interface. The excitation of a SPP induces a minimum in the specular reflectance spectra of $P$-polarized EM waves whose spectral position, in a first-order approximation, only depends of the dielectric constant and the period of the grating.

Both surface-shape resonances and SPPs have been comprehensively studied in metallic slabs with two-dimensional (2D) perforations in order to understand the phenomenon of extraordinary optical transmission (EOT) observed by Ebbesen and coworkers in 1998 [6]. In particular, it was shown that coaxial- or annular-type cavities present a superenhancement of the transmission due to the transverse electromagnetic (TEM) mode always present in this particular type of cavity [7-11].

Recently, 2D metallic gratings have been proposed as high-absorbing surfaces by embedding holes with lossy dielectrics [12,13]. Moreover, the advent of artificial structures, named metamaterials (MMs), with the ability to create independent tailored electric and magnetic responses has

\footnotetext{
*Current address: Institut d'Electronique, de Microlectronique et de Nanotechnologie, UMR CNRS 8520, Université de Lille 1, 59655 Villeneuve dAscq, France.

†jsdehesa@upv.es
}

boosted the investigation of novel physical phenomena with the promise of many interesting applications [14,15]. It is the microstructured arrangement of small-scale elements that drives the interaction of EM waves with these composites, thus providing true effective media with behaviors that can be tailored almost without limitation. This research field has reported, for example, the design and demonstration of absorbing metasurfaces with near-unity absorbance in $\mathrm{GHz}$ [16] and $\mathrm{THz}[17,18]$ regimes. The designed structures, which consisted of lossy MM subwavelength resonators periodically distributed on top of a dielectric slab, provided absorptivity close to $100 \%$ over a narrow frequency range.

This work presents the design and comprehensive analysis of high-absorbing structures consisting of a thin slab of a lossy dielectric on top of a metasurface. The metasurface is made of annular-type cavities patterned on a metallic plate. The plate is here considered as a perfect conductor and, therefore, the present study is valid at microwave frequencies. As in the MM approach, the subwavelength cavities provide the resonances, giving a strong concentration of EM energy which here is absorbed by the thin dielectric slab on top. Although annularcavity arrays have previously been studied with regards to their transmission characteristics [7-10,19], no studies have been performed exploring their use as building blocks for absorption enhancement. The fundamental mode (TEM mode) of the annular cavities has no cutoff frequency, a feature that is here employed to obtain extraordinary absorption at low frequencies. This is an advantage in comparison with empty-cavity designs where resonances are limited by the frequency cutoff determined by the cavity diameter. In comparison with metamaterial absorbers based on the repetition of metallic resonators [16], the absorption in our structures takes place at the dielectric absorbing layer on top on the metallic surface. This feature can be also considered as an advantage because of its easy fabrication; that is, the thin dielectric film is just deposited on top of the surface whereas the metallic resonators require a complex design together with very accurate fabrication. Also, the possibility of generating tailored surface dispersion characteristics to favor the coupling of the incident wave to surface waves is important for the objective of further enhancing the absorptivity $[11,20]$ 
In this study, the mode-matching technique is employed to rigorously analyze the interaction of a plane wave impinging on a thin and lossy dielectric layer backed by the metallic metasurface. Numerical simulations reveal the occurrence of several absorption regimes that can be associated with different features of the arrays. In particular, near-unity absorption can be achieved at long wavelengths.

The paper is organized as follows: After this introduction, Sec. II introduces the structures under study and describes the model approach employed in their analysis. Particularly, the mode-matching technique is briefly explained and applied to derive the analytical formulas giving the frequency-dependent absorbance $A(\omega)$. Full-wave simulations are also reported giving support to the model developed here. Afterwards, in Sec. III, we critically discuss the absorptive properties of the structures at low frequencies; i.e., below the diffraction limit. The different features of the absorptive peaks are comprehensively analyzed as a function of the structure parameters. Section IV studies other physical mechanisms of energy absorption, including the use of several cavities in the unit cell and the excitation of guided modes in the dielectric slab. Finally, Sec. V summarizes the main findings of this work and proposes some tasks for the future.

\section{MODEL AND NUMERICAL SIMULATIONS}

The structure under study is shown schematically in Fig. 1. The EM waves propagating in air (region I) impinge on a thin dielectric slab (region II) placed on top of a metallic metasurface (region III) containing a 2D array of annular cavities with length $h$ and inner and outer radii $r_{i}$ and $r_{e}$, respectively. The cavities are filled with a lossless dielectric material with permittivity $\varepsilon_{h}$ and are distributed in a square lattice with period $a$. The dielectric slab with complex dielectric constant $\hat{\varepsilon}_{d}=\varepsilon_{d}(1+i \xi)$ has thickness $\ell$, which will be considered very small in comparison either with $a$ as well as with the wavelength of the impinging waves (i.e., $\ell \ll a, \lambda$ ).

This section is divided into two parts. In the first part we develop an approximate model describing the low-frequency

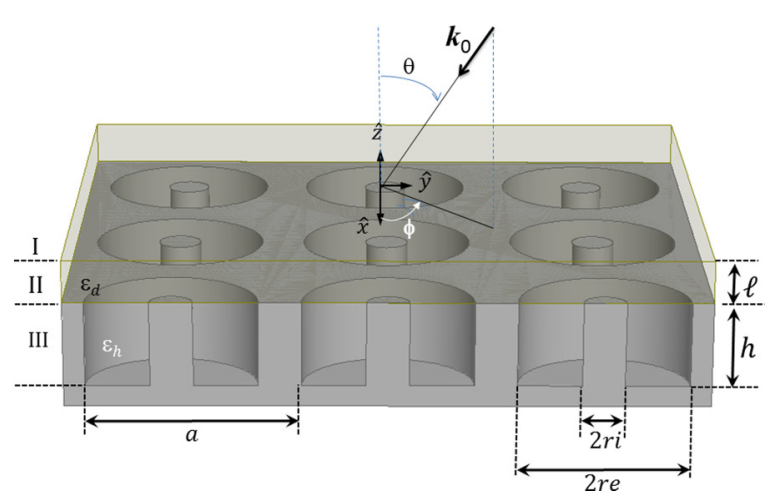

FIG. 1. (Color online) Schematic of proposed structures. Region I is the background medium (air in our case), region II is a dielectric slab with thickness $\ell$ and dielectric permittivity $\varepsilon_{d}(1+i \xi)$. Region III is a perfectly conducting metal containing a square distribution of annular cavities with lattice period $a$ and length $h$ filled with dielectric material $\varepsilon_{h}$. The external and inner radii of the annular cavities are $r_{e}$ and $r_{i}$, respectively. absorption of the structure, which is the topic of interest here. In the second part we report a kind of "numerical experiments" that are based on full-wave simulations using finite elements. The results from these simulations confirm the validity of the approximations employed in the analytical model and support the predictions here obtained.

\section{A. Analytical model}

We describe here the model giving the analytical expression for the absorbance $A(\omega)$, which is obtained from the difference between the outgoing and the incoming energy fluxes. Thus, $A(\omega)=1-\mathcal{R}(\omega)$, where $\mathcal{R}(\omega)$ is the reflectance of the full structure, and where region III is here considered as a perfect conducting metal.

It is assumed that the structure is illuminated (see Fig. 1) by a plane wave with wave vector $\boldsymbol{k}_{0}=(\omega / c)(\sin \theta \cos \phi \hat{\boldsymbol{x}}+$ $\cos \theta \sin \phi \hat{\boldsymbol{y}}+\cos \theta \hat{\boldsymbol{z}}$ ) (in spherical coordinates). The geometry of the problem indicates that the EM fields in the background and in the dielectric layer can be decomposed into components tangential and perpendicular to the metallic surface, which defines the $x y$ plane at $z=0$. Thus, the electric and magnetic fields are given by $\boldsymbol{E}(\mathbf{r})=E_{z} \hat{z}+\boldsymbol{E}_{t}$ and $\boldsymbol{B}(\mathbf{r})=B_{z} \hat{z}+\boldsymbol{B}_{t}$, respectively, and the wave vector can be expressed as $\boldsymbol{k}_{0}=q_{0} \hat{z}+\boldsymbol{k}_{t}$, such that $k_{0}^{2}=q_{0}^{2}+\left|\boldsymbol{k}_{t}\right|^{2}$. The light polarization $\sigma$ is named $S$ when $\boldsymbol{E}_{t} \perp \boldsymbol{k}_{t}$ or $P$ when $\boldsymbol{E}_{t} \| \boldsymbol{k}_{t}$. The temporal dependence $e^{-i \omega t}$ will be implicitly assumed in the rest of the paper for all fields.

The interaction of the EM waves with the $2 \mathrm{D}$ array of subwavelength cavities will excite two kinds of resonant modes: (1) modes localized inside the cavities and (2) surface waves with tangential wave number $\boldsymbol{k}_{G}=\boldsymbol{k}_{t}+\boldsymbol{G}$, where $\boldsymbol{G}$ are the reciprocal lattice vectors

$$
\boldsymbol{G}=m_{1} \mathbf{b}_{1}+m_{2} \mathbf{b}_{2},
$$

where $\mathbf{b}_{i}$ are the primitive vectors of the reciprocal lattice [21]. A square lattice is here comprehensively studied but results can be equally applied to a hexagonal lattice.

The solution for $\boldsymbol{E}_{t}$ and $\boldsymbol{H}_{t}$ can be obtained as a linear combination of these diffracted waves, which additionally can be decomposed into two polarizations $\sigma=S, P$, with $S$ and $P$ being the polarizations perpendicular and parallel, respectively, to the wave vector $\boldsymbol{k}_{G}$, and whose unit vectors are given by

$$
\hat{\boldsymbol{u}}_{G S}=\frac{\hat{\boldsymbol{z}} \times \boldsymbol{k}_{G}}{\left|\boldsymbol{k}_{G}\right|}
$$

for the $S$ polarization and

$$
\hat{\boldsymbol{u}}_{G P}=\frac{\boldsymbol{k}_{G}}{\left|\boldsymbol{k}_{G}\right|}
$$

for the $P$ polarization.

Therefore, the fields in region I (the air) are expressed as

$$
\begin{aligned}
\left|\boldsymbol{E}_{t}^{0}\right\rangle= & A_{0 \sigma_{0}}^{-} e^{-i q_{0}(z-\ell)}\left|\boldsymbol{k}_{t} \sigma_{0}\right\rangle+\sum_{G, \sigma} A_{G \sigma}^{+} e^{i q_{G}(z-\ell)}\left|\boldsymbol{k}_{G} \sigma\right\rangle, \\
\left|-\hat{z} \times \boldsymbol{H}_{t}^{0}\right\rangle= & -Y_{0 \sigma_{0}}^{0} A_{0 \sigma_{0}}^{-} e^{-i q_{0}(z-\ell)}\left|\boldsymbol{k}_{t} \sigma_{0}\right\rangle \\
& +\sum_{G, \sigma} Y_{G \sigma}^{0} A_{G \sigma}^{+} e^{i q_{G}(z-\ell)}\left|\boldsymbol{k}_{G} \sigma\right\rangle,
\end{aligned}
$$


where $A_{0 \sigma_{0}}^{-}$is the amplitude of the incident wave with polarization $\sigma_{0}$ and $q_{G}^{2}=\left(\omega / c_{0}\right)^{2}-\left|\boldsymbol{k}_{G}\right|^{2}$.

Using Dirac's notation, the diffracted wave with wave vector $\boldsymbol{k}_{G}$ and polarization $\sigma$ is denoted by the ket $\left|\boldsymbol{k}_{G} \sigma\right\rangle$, and their normalized expressions are

$$
\left\langle\boldsymbol{r} \mid \boldsymbol{k}_{G \sigma}\right\rangle \equiv \frac{1}{\sqrt{\Omega}} e^{i \boldsymbol{k}_{G} \cdot \boldsymbol{r}} \hat{\boldsymbol{u}}_{\boldsymbol{G} \sigma},
$$

where $\Omega$ is the area of the unit cell and they satisfy $\left\langle\boldsymbol{k}_{G} \sigma \mid r\right\rangle\left\langle r \mid \boldsymbol{k}_{G} \sigma\right\rangle=1$.

The modal admittances $Y_{G \sigma}$ for the two polarizations are

$$
Y_{G S}^{0}=\frac{q_{G}}{k_{\omega}} \sqrt{\frac{\varepsilon_{0}}{\mu_{0}}},
$$

and

$$
Y_{G P}^{0}=\frac{k_{\omega}}{q_{G}} \sqrt{\frac{\varepsilon_{0}}{\mu_{0}}},
$$

where $\varepsilon_{0}$ and $\mu_{0}$ are, respectively, the dielectric permittivity and magnetic permeability of air.

Similarly, the tangential components of the fields in region II (the dielectric slab) are

$$
\left|\boldsymbol{E}_{t}^{d}\right\rangle=\sum_{G, \sigma}\left(B_{G \sigma}^{+} e^{i p_{G}(z-\ell)}+B_{G \sigma}^{-} e^{-i p_{G}(z-\ell)}\right)\left|\boldsymbol{k}_{G} \sigma\right\rangle,
$$

and

$$
\begin{aligned}
\left|-\hat{z} \times \boldsymbol{H}_{t}^{d}\right\rangle= & \sum_{G, \sigma} Y_{G \sigma}^{d}\left(B_{G \sigma}^{+} e^{i p_{G}(z-\ell)}\right. \\
& \left.-B_{G \sigma}^{-} e^{-i p_{G}(z-\ell)}\right)\left|\boldsymbol{k}_{G} \sigma\right\rangle,
\end{aligned}
$$

with $p_{G}^{2}=k_{d}^{2}-\left|\boldsymbol{k}_{G}\right|^{2}$ and $k_{d}=k_{0} \sqrt{\varepsilon_{d} \mu_{d}}$.

The modal admittances for each polarization are now

$$
Y_{G S}^{d}=\frac{p_{G}}{k_{\omega}} \sqrt{\frac{\varepsilon_{d}}{\mu_{d}}},
$$

and

$$
Y_{G P}^{d}=\frac{k_{\omega}}{p_{G}} \sqrt{\frac{\varepsilon_{d}}{\mu_{d}}} .
$$

Finally, for the fields in region III (the metal with the cavity array) we employ a modal expansion of the electric and magnetic fields inside the cavities. It is well known that the resonant frequencies inside the annular cavity are characterized by $M$, the azimuthal mode number (half the number of nodes) around the circumference of the annulus, and $N$, the longitudinal mode number. A rigorous calculation of the modes inside a single cavity can be performed using Bessel functions of the first kind [22]. For the present discussion, we may write the resonant frequencies (ignoring end effects) approximately as [8]

$$
\omega_{(M, N)}=c\left[\left(\frac{2 M}{r_{i}+r_{e}}\right)^{2}+\left(\frac{N \pi}{h}\right)^{2}\right]^{\frac{1}{2}},
$$

which is obtained with the condition of a perfect conductor and is a good approximation when $r_{i}$ is comparable in magnitude to $r_{e}$.

In the present work our model considers the family of modes with $M=0$ in the modal expansion inside the cavities, which is justified in the limit where the light wavelength is much larger than the diameter of the cavities. These modes are excited only with radiation incident off normal.

The fundamental mode $|\alpha\rangle$ is the TEM mode of a coaxial waveguide, which has no cutoff frequency, and the fields inside the cavities are

$$
\begin{gathered}
\left|\boldsymbol{E}_{t}^{\alpha}\right\rangle=C_{\alpha}\left(e^{i k_{h} z}+\Gamma_{\alpha} e^{-i k_{h} z}\right)|\alpha\rangle, \\
\left|-\hat{z} \times \boldsymbol{H}_{t}^{\alpha}\right\rangle=Y_{\alpha} C_{\alpha}\left(e^{i k_{h} z}-\Gamma_{\alpha} e^{-i k_{h} z}\right)|\alpha\rangle,
\end{gathered}
$$

where the modal admittance is $Y_{\alpha}=\sqrt{\varepsilon_{h} / \mu_{h}}$ and the reflection coefficient at the cavity bottom is $\Gamma_{\alpha}=-e^{-2 i k_{h} h}$. The normalized TEM mode is

$$
\langle\mathbf{r} \mid \alpha\rangle=-\frac{1}{\sqrt{2 \pi}} \frac{e^{i \boldsymbol{k}_{G} \cdot \boldsymbol{R}_{\alpha}}}{\sqrt{\ln \left(r_{e} / r_{i}\right)}} \frac{1}{r} \hat{\boldsymbol{r}} .
$$

At the air-dielectric interface, located at $z=\ell$, the continuity of the tangential components of the fields gives

$$
\begin{gathered}
A_{G \sigma}^{+}+A_{0 \sigma_{0}}^{-} \delta_{0 \sigma_{0}}=B_{G \sigma}^{+}+B_{G \sigma}^{-}, \\
Y_{G \sigma}^{0}\left(A_{G \sigma}^{+}-A_{0 \sigma_{0}}^{-} \delta_{0 \sigma_{0}}\right)=Y_{G \sigma}^{d}\left(B_{G \sigma}^{+}-B_{G \sigma}^{-}\right) .
\end{gathered}
$$

At $z=0$, the metal-dielectric interface, the boundary conditions impose the continuity of $\boldsymbol{E}_{t}$ over the entire unit cell and the continuity of $\boldsymbol{H}_{t}$ over the annular cavity. Thus, at the interface the field equations are

$$
\begin{aligned}
& \sum_{G, \sigma}\left(B_{G \sigma}^{+} e^{-i p_{G} \ell}+B_{G \sigma}^{-} e^{i p_{G} \ell}\right)\left|\boldsymbol{k}_{G} \sigma\right\rangle=C_{\alpha}\left(1+\Gamma_{\alpha}\right)|\alpha\rangle, \\
& \sum_{G, \sigma} Y_{G \sigma}^{d}\left(B_{G \sigma}^{+} e^{-i p_{G} \ell}-B_{G \sigma}^{-} e^{i p_{G} \ell}\right)\left|\boldsymbol{k}_{G} \sigma\right\rangle=Y_{\alpha} C_{\alpha}\left(1-\Gamma_{\alpha}\right)|\alpha\rangle .
\end{aligned}
$$

Now, Eq. (19) is projected with the mode $\left\langle\boldsymbol{k}_{G} \sigma\right|$ while Eq. (20) is projected with cavity mode $\langle\alpha|$, leading to

$$
\begin{gathered}
B_{G \sigma}^{+} e^{-i p_{G} \ell}+B_{G \sigma}^{-} e^{i p_{G} \ell}=C_{\alpha}\left(1+\Gamma_{\alpha}\right)\left\langle\boldsymbol{k}_{G} \sigma \mid \alpha\right\rangle, \\
\sum_{G, \sigma} Y_{G \sigma}^{d}\left(B_{G \sigma}^{+} e^{-i p_{G} \ell}-B_{G \sigma}^{-} e^{i p_{G} \ell}\right)\left\langle\alpha \mid \boldsymbol{k}_{G} \sigma\right\rangle=Y_{\alpha} C_{\alpha}\left(1-\Gamma_{\alpha}\right) .
\end{gathered}
$$

The term $\left\langle\alpha \mid \boldsymbol{k}_{G} \sigma\right\rangle=\left\langle\boldsymbol{k}_{G} \sigma \mid \alpha\right\rangle^{*}$ represents the overlap integrals of the diffracted waves in the dielectric layer with the TEM mode inside the annular cavities. For $S$-polarized waves the integral is canceled, $\left\langle\alpha \mid \boldsymbol{k}_{G} S\right\rangle=0$, while for $P$-polarized waves the integral takes the following form (see Appendix A):

$$
\begin{aligned}
\left\langle\boldsymbol{k}_{G} P \mid \alpha\right\rangle= & \left\langle\alpha \mid \boldsymbol{k}_{G} P\right\rangle^{*}=-i \frac{\sqrt{2 \pi} b}{\sqrt{\Omega}} \frac{e^{i \boldsymbol{k}_{G} \cdot \boldsymbol{R}_{\alpha}}}{\sqrt{\ln \left(r_{e} / r_{i}\right)}} \\
& \times \frac{J_{0}\left(k_{G} r_{e}\right)-J_{0}\left(k_{G} r_{i}\right)}{k_{G} r_{e}} .
\end{aligned}
$$

The unknown field coefficients in each region are finally obtained by combining Eqs. (17), (18), (21), and (22).

The coefficients $C_{\alpha}$ inside the cavities are

$$
C_{\alpha}=-\frac{4}{M} \frac{Y_{0}^{0} Y_{0}^{d}}{Y_{0}^{H} Y_{\alpha}}\left\langle\alpha \mid \boldsymbol{k}_{t} P\right\rangle e^{i p_{0} \ell} A_{0}^{-},
$$


with

$$
Y_{G}^{H}=Y_{G P}^{0}\left(1-e^{2 i p_{G} \ell}\right)+Y_{G P}^{d}\left(1+e^{2 i p_{G} \ell}\right),
$$

and

$$
\begin{gathered}
M=\left(1-\Gamma_{\alpha}\right)-\left(1+\Gamma_{\alpha}\right) \chi \\
\chi=\sum_{G} H_{G G}^{2} \frac{Y_{G P}^{d}}{Y_{\alpha}} \frac{Y_{G P}^{0} \cos \left(p_{G} \ell\right)-i Y_{G P}^{d} \sin \left(p_{G} \ell\right)}{-i Y_{G P}^{0} \sin \left(p_{G} \ell\right)+Y_{G P}^{d} \cos \left(p_{G} \ell\right)}
\end{gathered}
$$

with

$$
H_{G G}^{2}=\left\langle\alpha \mid \boldsymbol{k}_{G} P\right\rangle\left\langle\boldsymbol{k}_{G} P \mid \alpha\right\rangle=\left|\left\langle\boldsymbol{k}_{G} P \mid \alpha\right\rangle\right|^{2} .
$$

For the coefficients in the dielectric layer (region II) we get

$$
\begin{aligned}
B_{G P}^{-}= & 2 \frac{Y_{G P}^{0}}{Y_{G}^{H}} A_{0}^{-} \delta_{G, 0}+\frac{Y_{G P}^{d}-Y_{G P}^{0}}{Y_{G}^{H}} C_{\alpha}\left(1+\Gamma_{\alpha}\right) \\
& \times\left\langle\boldsymbol{k}_{G} P \mid 0\right\rangle e^{i p_{G} \ell} \\
B_{G P}^{+}= & C_{\alpha}\left(1+\Gamma_{\alpha}\right)\left\langle\boldsymbol{k}_{G} P \mid 0\right\rangle e^{i p_{G} \ell}-B_{G P}^{-} e^{2 i p_{G} \ell} .
\end{aligned}
$$

And the coefficients in the air (region I) are

$$
\begin{aligned}
A_{G P}^{+}= & \left(R_{d} \delta_{G 0}-8 \frac{Y_{0 P}^{0} Y_{0 P}^{d}}{Y_{0}^{H} Y_{\alpha}} \frac{Y_{G P}^{d}}{Y_{G}^{H}} \frac{\left(1+\Gamma_{\alpha}\right)}{M}\right. \\
& \left.\times e^{i p_{0} \ell} e^{i p_{G} \ell}\left\langle\alpha \mid \boldsymbol{k}_{t} P\right\rangle\left\langle\boldsymbol{k}_{G} P \mid \alpha\right\rangle\right) A_{0}^{-},
\end{aligned}
$$

where $R_{d}$ is the reflection coefficient of the dielectric layer without the metallic surface:

$$
R_{d}=\frac{i Y_{0 P}^{0} \sin \left(p_{0} \ell\right)+Y_{0 P}^{d} \cos \left(p_{0} \ell\right)}{i Y_{0 P}^{0} \sin \left(p_{0} \ell\right)-Y_{0 P}^{d} \cos \left(p_{0} \ell\right)} .
$$

Once the fields are determined in the three regions, the reflectance of the system is calculated in terms of $A_{G^{\prime} P}^{+}$, which is the amplitude of the reflected waves in the background.

Let us remark that the overlap integral $\left\langle\alpha \mid \boldsymbol{k}_{\boldsymbol{t}} P\right\rangle$ appearing in Eq. (31) becomes zero at normal incidence; i.e., $\langle\alpha \mid \mathbf{0} P\rangle=0$ [see Eq. (16)]. As a consequence, the reflection coefficients in the air reduce to the reflection coefficient of the dielectric layer; $A_{G P}^{+}=R_{d} \delta_{G, 0}$, for any wave vector $G$.

In this work our interest is focused on frequencies below the diffraction limit and, therefore, we consider that only the fundamental mode $\boldsymbol{G}=0$ is excited. In other words, the reflection coefficient is simply

$$
R_{0}(\omega)=A_{0 P}^{+} / A_{0}^{-}
$$

and the absorbance in the dielectric slab is

$$
A(\omega)=1-\left|R_{0}(\omega)\right|^{2} .
$$

Figure 2(a) shows a typical absorbance spectrum calculated for incident EM waves with wave vector defined by the angles $\phi=0$ and $\theta=\pi / 4$. The dielectric slab with thickness $\ell=$ $0.3 a$ has complex permittivity $\hat{\varepsilon}_{d}=2+i 0.05$, and the metallic metasurface consists of a square distribution of annular cavities with length $h=a$ and radii $r_{i}=0.1 a, r_{e}=0.4 a$. The dotted lines in this figure represent the absorptance calculated for a dielectric layer without the annular cavities in the metal.
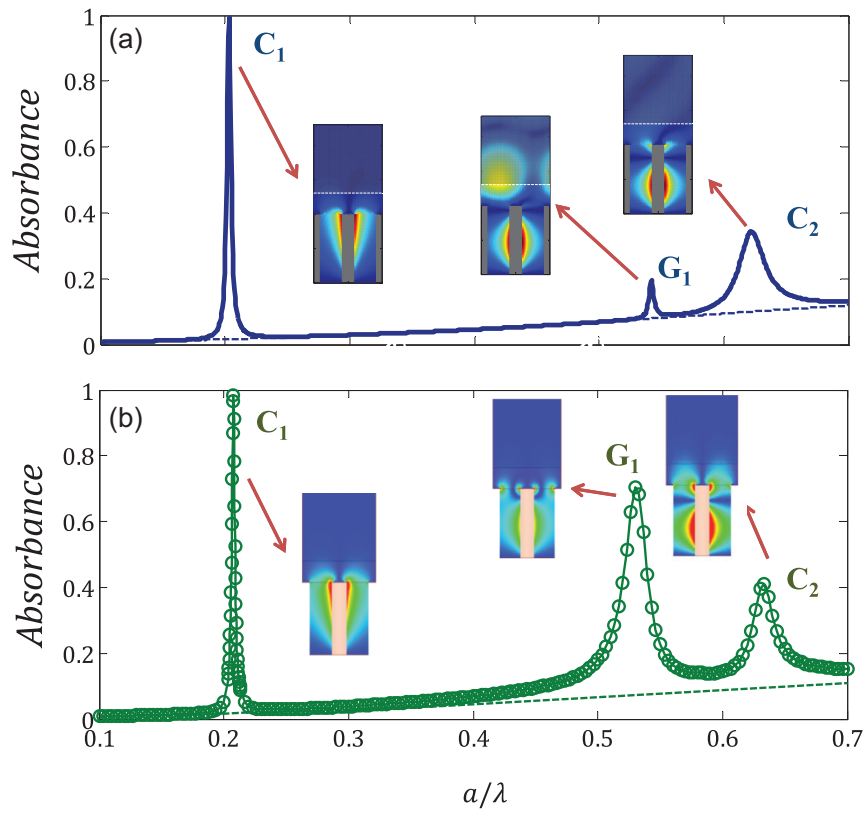

FIG. 2. (Color online) Absorbance spectrum $A(\omega)$ obtained for the structure schematically described in Fig. 1. Results are obtained with a $P$-polarized plane wave impinging along the direction $\phi=0$ and $\theta=\pi / 4$. The slab with thickness $\ell / a=0.3$ is a lossy dielectric with permittivity $\varepsilon_{d}=2+i 0.05$. The metallic surface contains a square distribution of annular cavities with radii $r_{i} / a=0.1, r_{e} / a=$ 0.4 , and length $h / a=1$. The dashed curve corresponds to the absorbance of the slab with a flat metal underneath, that shows the same result at normal incidence $(\theta=0)$. The insets depict the fields at the peak positions. (a) Theoretical simulation. (b) HFSS simulation.

The results in Fig. 2(a) lead us to conclude that the array of cavities is responsible for the absorptive peaks. In brief, two kind of peaks are clearly distinguished. The peaks $C_{1}$ and $C_{2}$ appear at frequencies corresponding to coaxial cavity resonances; the insets show that $C_{1}$ corresponds to the fundamental TEM mode while $C_{2}$ represents the (Fabry-Perot) higher order TEM mode. Note that the lossy slab perfectly absorbs the EM energy localized in the TEM mode $C_{1}$. In between, the low absorptive peak $G_{1}$ corresponds to a hybrid mode; a combination of standing waves localized in the cavity with a propagating guided mode in the slab.

\section{B. Numerical experiments}

In order to support the results obtained from the analytical model, full-wave simulations have been performed by using a commercial software based on finite elements [23]. The corresponding results are shown in Fig. 2(b) and demonstrate that the absorptive profiles of the fundamental mode $C_{1}$ and its first higher mode $C_{2}$ are equal to those obtained from the model [see Fig. 2(a)].

However, it is observed that the absorptive profile of peak $G_{1}$, corresponding to the hybrid mode, is not well reproduced since the model only takes into account the fundamental TEM mode and its corresponding Fabry-Perot-like modes quantized by the cavity length $h$. We should remark that $G_{1}$ is above the cutoff frequency of the grating modes due to the periodicity of the lattice. The possible interaction of this type of mode with 
the evanescent modes in the coaxial cavity cannot be precisely modeled with our method. This is obvious since our model only considers the fundamental mode, the one with azimuthal mode number $M=0$, and its $N$ longitudinal excitations inside the coaxial cavities. Despite this slight disagreement, our model gives a good quantitative prediction of the peak positions at which the structure absorbs energy.

In the following sections we focus our analysis on the behavior of the low-frequency peak of type $C_{1}$, for which the model perfectly reproduces the full-wave simulations. Nevertheless, additional considerations will be given about the guided modes of the slab in order to get a physical insight into the origin of the $G_{1}$ absorption peaks.

\section{LOW-FREQUENCY ABSORPTION: RESULTS AND DISCUSSION}

This section comprehensively studies the low-frequency absorptive peak $C_{1}$ shown in Fig. 2. Note that this peak appears below the diffraction limit, where only the fundamental mode is excited in the metallic metasurface. It is also noticeable that the peak appears at a wavelength $\lambda_{1} \approx 5 a=15 \ell$. These features are employed below in order to obtain the reflection coefficient $R_{0}(\omega)$ and the absorptance $A(\omega)$ given by Eqs. (33) and (34), respectively.

For a thin dielectric slab we can assume that $p_{0} \ell \ll 1$, then $R_{d} \approx-1$ and it is easy to show from Eq. (31) that the reflection coefficient can simplified to

$$
R_{0}=-\frac{\cos k_{h} h+i\left(H_{00}^{2}+\chi\right) \sin k_{h} h}{\cos k_{h} h-i \chi \sin k_{h} h},
$$

where $H_{00}^{2}=\left\langle\alpha \mid \boldsymbol{k}_{t} P\right\rangle\left\langle\boldsymbol{k}_{t} P \mid \alpha\right\rangle$.

The lattice sum term $\chi$ given in Eq. (27) is a frequencydependent complex quantity that can be expressed as $\chi(\omega)=$ $\chi_{R}+i \chi_{I}$. Therefore, the reflection coefficient can be given as

$$
R_{0}=-\frac{\cos k_{h} h+\chi_{I} \sin k_{h} h+i\left(H_{00}^{2}-\chi_{R}\right) \sin k_{h} h}{\cos k_{h} h+\chi_{I} \sin k_{h} h-i \chi_{R} \sin k_{h} h},
$$

and the absorbance of the system is

$$
A(\omega)=1-\frac{\left(\cot k_{h} h+\chi_{I}\right)^{2}+\left(H_{00}^{2}-\chi_{R}\right)^{2}}{\left(\cot k_{h} h+\chi_{I}\right)^{2}+\chi_{R}^{2}} .
$$

The behavior in frequency of $\chi_{I}$ and $\chi_{R}$ is obtained by splitting the lattice sum in $\chi$ in the propagating $\left(q_{G}\right.$ and $p_{G}$ real) and evanescent contributions $\left(q_{G}\right.$ and $p_{G}$ imaginary). Since we are below the diffraction limit in both the dielectric and the background, the only propagating mode is that with $\boldsymbol{G}=0$. Thus,

$$
\chi(\omega)=\chi_{0}+\sum_{G \neq 0} \chi_{G},
$$

where the term $\chi_{0}$ is obtained from Eq. (27) and is given by

$$
\begin{aligned}
\chi_{0} & =\frac{Y_{0 P}^{d}}{Y_{\alpha}} \frac{Y_{0 P}^{0} \cos \left(p_{0} \ell\right)-i Y_{0 P}^{d} \sin \left(p_{0} \ell\right)+Y_{0 P}^{d} \sin \left(p_{0} \ell\right)}{\left.-i p_{0} \ell\right)} H_{00}^{2} \\
& \approx \frac{k_{\omega}}{\sqrt{k_{\omega}^{2}-\left|k_{t}\right|^{2}}} \sqrt{\frac{\varepsilon_{0}}{\varepsilon_{h}}} H_{00}^{2} ;
\end{aligned}
$$

the last expression being obtained with the approximation $\sin p_{0} \ell \approx 0$. Note that $\chi_{0}$ is real since there are no losses either in the background or in the cavities.

The terms $\chi_{G}$ for $\boldsymbol{G} \neq 0$ cannot be simplified with the same approach since the lattice sum is infinite and the product $p_{G} \ell$ grows linearly. Also, the presence of the complex permittivity of the dielectric slab in the expressions makes it difficult to split these terms into their real and imaginary parts. To do it, we make a Taylor expansion of these terms around $\xi=0$,

$$
\chi_{G}(\xi) \approx \chi_{G}(\xi=0)+\xi \partial_{\xi} \chi_{G}(\xi=0) .
$$

In this expansion, we know that $\chi_{G}(\xi=0)$ is purely imaginary and $\partial_{\xi} \chi_{G}(\xi=0)$ is real (see Appendix B). Therefore,

$$
\begin{aligned}
& \chi_{R}(\omega) \approx \chi_{0}+\xi \chi_{G}^{\prime}(\xi=0), \\
& \chi_{I}(\omega) \approx-i \chi_{G}(\xi=0) .
\end{aligned}
$$

The frequency dependence of both $\chi_{R}(\omega)$ and $H_{00}(\omega)$ is very smooth. Then it can be assumed that both are constant at low frequencies. On the contrary, it can be seen that the frequency dependence of $\chi_{I}$ is predominantly linear in $k_{\omega}$ with negative slope; that is,

$$
\chi_{I}=-i \chi_{G}(\xi=0) \sim-i \frac{Y_{G}^{d}}{Y_{\alpha}} \sim-k_{\omega},
$$

since we are under the condition $\boldsymbol{G} \neq 0$ and $p_{G}$ is purely imaginary.

The above considerations lead us to conclude that the frequency dependence of the absorbance is

$$
A(\omega)=1-\frac{f^{2}(\omega)+c_{1}^{2}}{f^{2}(\omega)+c_{2}^{2}},
$$

where $f(\omega)^{2}=\left(\cot k_{h} h+\chi_{I}\right)^{2} . A(\omega)$ has a maximum as long as $f(\omega) f^{\prime}(\omega)=0$. But $f^{\prime}(\omega)$ is negative for the frequency region of interest and, therefore, the only possibility of having a maximum is $f(\omega)=0$. So the condition for having a peak in the absorbance is

$$
\cot k_{h} h+\chi_{I}(\omega)=0 .
$$

In fact, this expression relates the peak position with the cavity length $h$ and the lattice sum $\chi_{I}$, which contains only the contribution of the evanescent modes. Therefore, it is concluded that the position of the peak is mainly determined by the interaction of the evanescent waves in the dielectric and the fundamental mode of the annular cavities. Also, it must be pointed out that the above condition depends on the layer's thickness $\ell$, but it is independent of $\xi$, the dissipative term of the permittivity.

The amplitude of the absorbance has a frequency dependence that can be obtained from Eq. (36). When this amplitude fulfills the condition (44), the peak amplitude only depends on the term $H_{00}^{2}-\chi_{R}$, which gives perfect absorption (unity amplitude) when its value is zero. In other words, the condition for having perfect absorption is

$$
H_{00}^{2}-\chi_{R}=0 .
$$

This condition shows that perfect absorption depends on the value for $\xi$ through $\chi_{R}$, but it is independent of $h$. Interestingly, the condition for perfect absorption is independent of the 

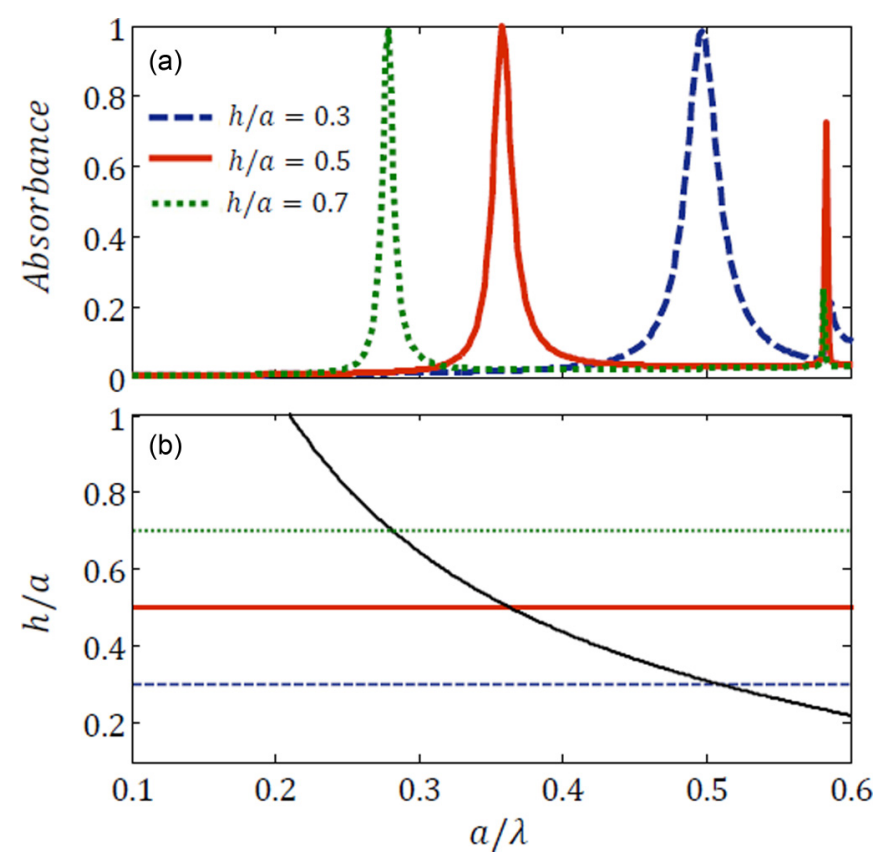

FIG. 3. (Color online) (a) Absorbance spectra for three values of cavity length $h$. (b) Frequency of the absorptive peak as a function of $h$ (black line). Results are obtained by using the incident direction $\theta=\pi / 4, \phi=0$ and the following parameters of the structure: $r_{i} / a=$ $0.1, r_{e} / a=0.4, \ell / a=0.1, \varepsilon_{d}=2+i 0.1$, and $\varepsilon_{h}=1$.

frequency at which it occurs, since it has been already mentioned that the dependence in frequency of $H_{00}$ and $\chi_{R}$ is smooth.

Figure 3(a) depicts the behavior of the low-frequency peak for three values of the cavity length $h$. Dashed, continuous, and dotted lines represent the results for $h / a=0.3,0.5$, and 0.8 , respectively. It is observed that the peak amplitude does not depend on $h$, but its profile becomes sharper for lower values of the frequency position. This behavior is strictly related to the fact that TEM modes in longer cavity depths have a weaker interaction with the dielectric slab. It is also clear that the position of the peak strongly depends on the length $h$. Moreover, this result indicates that the amplitude remains constant when the coaxial length changes. Figure 3(b) represents the condition (44) for having an absorption peak with the black line, and the horizontal dotted lines mark the coaxial lengths for which the absorption spectra are represented in the top panel. A clear correspondence is noticed between the crossing frequencies in Fig. 3(b) and the peak positions in Fig. 3(a).

Variation in the absorption properties with the outer and inner radius of the coaxial aperture is presented in Figs. 4 and 5. Note that the dependence on $r_{e}$ is also giving the dependence with the lattice filling fraction $f$, which is $f=\pi\left(r_{e} / a\right)^{2}$ for the square lattice. The peak position is slightly connected with the coaxial radii. In case of variations in $r_{e}$, the peak position goes down in frequency when $r_{e}$ increases; i.e., for larger cavities the TEM mode becomes more localized and decreases in frequency. On the other hand, when the inner radii $r_{i}$ increases the peak position moves to higher frequencies since the annular aperture becomes thinner. However, note
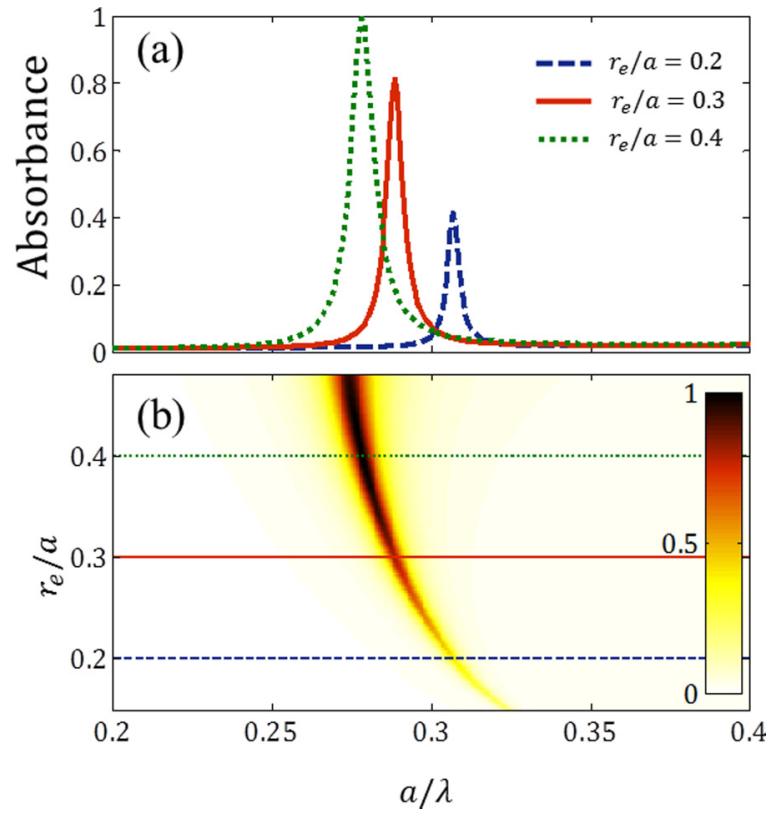

FIG. 4. (Color online) (a) Absorbance spectra $A(\omega)$ calculated for three values of the external cavity radius $r_{e}$. (b) Absorbance enhancement as a function of $r_{e}$. The structure dimensions are $r_{i} / a=0.1, h / a=0.7, \ell / a=0.1$, the permittivity is $\varepsilon_{d}=2+i 0.1$, and the incidence angles are $\theta=\pi / 4, \phi=0$.

that the peak amplitude increases with the outer radii but is independent of the inner radii.

Figure 6 shows how the absorption peaks changes in shape with the losses in the dielectric $\xi$, comparing the absorption spectrum for $\xi=0.025,0.05,0.1$, for a structure
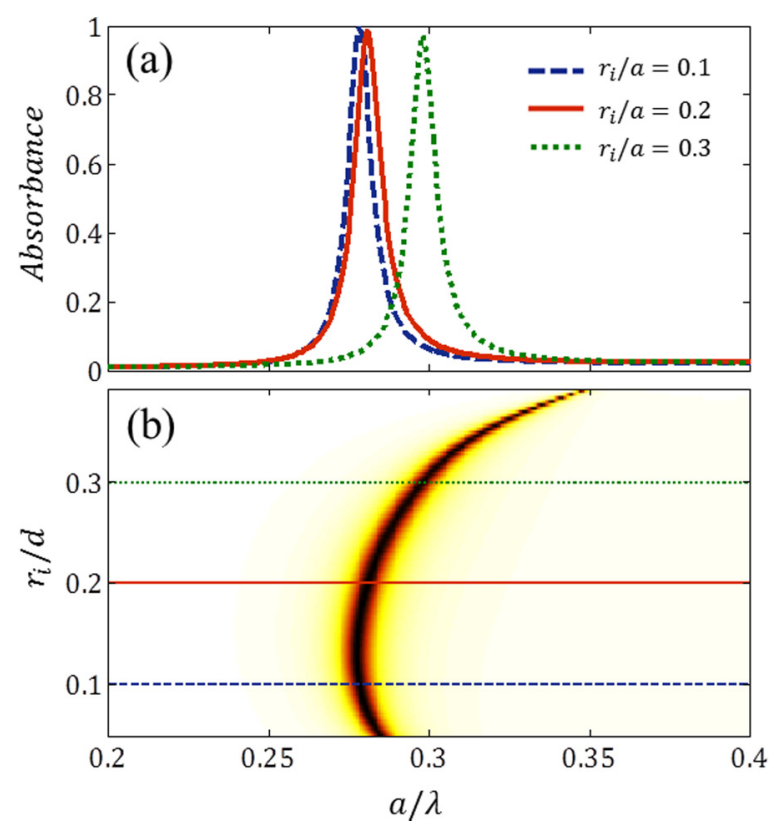

FIG. 5. (Color online) (a) Absorbance as a function of the annular inner radius $r_{i}$. The structure dimensions are $r_{e} / a=0.4, h / a=0.7$, $\ell / a=0.1$. The permittivity is $\varepsilon_{d}=2+i 0.1$ and the incidence angles are $\theta=\pi / 4, \phi=0$. (b) Absorbance enhancement in the active layer as a function of $r_{i}$. 


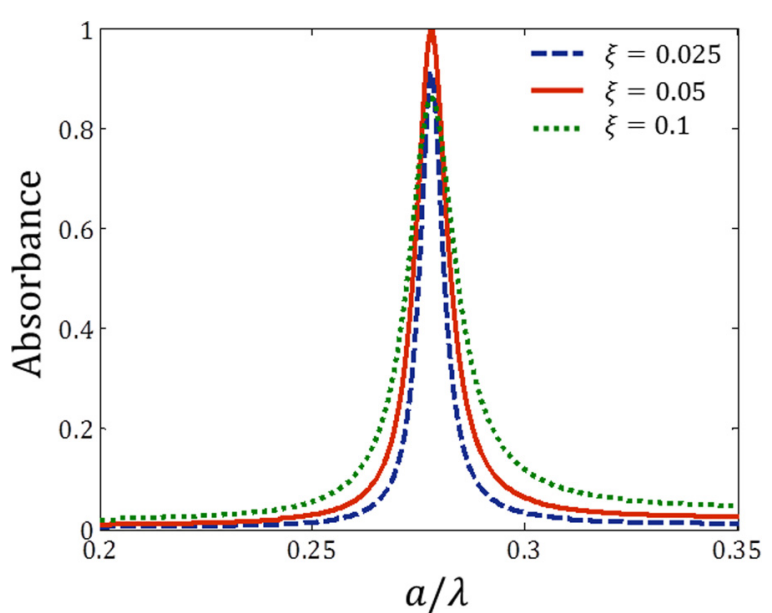

FIG. 6. (Color online) Absorbance in the dielectric slab with thickness $\ell / a=0.1$ for three values of $\xi$ in its dielectric permittivity $\varepsilon_{d}=2(1+i \xi)$. The annular cavities on the metallic metasurface have dimensions $r_{i} / a=0.1, r_{e} / a=0.4$, and $h / a=0.7$. Results are obtained for the incidence angles $\theta=\pi / 4$ and $\phi=0$.

with $r_{i} / a=0.1, r_{e} / a=0.4, h / a=0.7, \ell / a=0.1, \theta=\pi / 4$, and $\phi=0$. Note that the peak position does not depend on the dielectric losses in the slab. Note that perfect absorption (unity absorbance) is obtained for $\xi=0.05$ and not for the larger value $\xi=0.1$, indicating that $\xi$ should be optimized for a given structure in order to achieve perfect absorption.

Finally, Fig. 7 shows the absorbance for three thicknesses of the dielectric slab. Notice that amplitude of the peak is independent of the dielectric thickness, meaning that with the proper design perfect absorption is obtained independently of the thickness of the absorbing layer. However, also note that the peak profile becomes broader for larger thickness, indicating that absorption is proportional to the volume of the active layer.

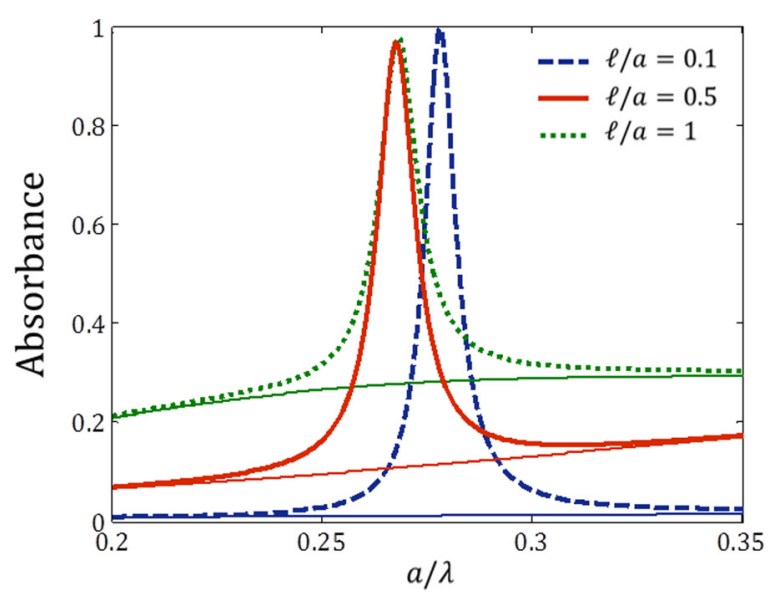

FIG. 7. (Color online) Absorbance of a dielectric slab with permittivity $\varepsilon_{d}=2+i 0.1$ for three different thicknesses. The annular cavities on the metallic surface have dimensions $r_{i} / a=0.1, r_{e} / a=$ $0.4, h / a=0.7$. Continuous lines under the peaks represent the absorbance without the metallic metasurface. Results are obtained for the incident angles $\theta=\pi / 4$ and $\phi=0$.

\section{OTHER ABSORPTION MECHANISMS}

We have learned from the previous section that the mechanism acting at low frequencies is easily tunable to get perfect absorption as a consequence of the interaction between the metallic metasurface and the thin absorbing slab. In this section two additional mechanisms of absorption by thin films will be studied and, although it will be shown that they are less efficient, they may offer more degrees of freedom to improve the absorption by the thin film.

The first mechanism considers the possibility of using a non-Bravais lattice; i.e., by considering more than one cavity in the unit cell. The second mechanism will take into account the excitation of guided modes in the slab by the metallic grating underneath.

\section{A. Non-Bravais lattices}

Let us consider now the case in which more than one cavity is associated with one point of a Bravais lattice. In other words, we study here a periodic arrangement of cavities with $N$ annular cavities inside the unit cell of the square lattice. The position of the $\alpha$ aperture in the unit cell is given by a vector $\boldsymbol{R}_{\alpha}$, with $\alpha=1,2, \ldots, N$. Under the monomode approximation we have now a set of $N$ coefficients $C_{\alpha}$, one for each cavity, then mode matching has to be applied at every aperture, and Eqs. (21) and (22) become

$$
\begin{gathered}
B_{G \sigma}^{+} e^{-i p_{G} \ell}+B_{G \sigma}^{-} e^{i p_{G} \ell}=\sum_{\beta} C_{\beta}\left(1+\Gamma_{\beta}\right)\left\langle\boldsymbol{k}_{G} \sigma \mid \beta\right\rangle, \\
\sum_{G, \sigma} Y_{G \sigma}^{d}\left(B_{G \sigma}^{+} e^{-i p_{G} \ell}-B_{G \sigma}^{-} e^{i p_{G} \ell}\right)|G \sigma\rangle\left\langle\alpha \mid \boldsymbol{k}_{G} \sigma\right\rangle \\
=Y_{\alpha} C_{\alpha}\left(1-\Gamma_{\alpha}\right) .
\end{gathered}
$$

By solving the system of equations defined by Eqs. (17), (18), (46), and (47), we get

$$
\begin{aligned}
A_{G P}^{+}= & \left(R_{0} \delta_{G, 0}-8 e^{i p_{0} \ell} e^{i p_{G} \ell} \frac{Y_{0 P}^{0} Y_{0 P}^{d}}{Y_{0}^{H}} \frac{Y_{G P}^{d}}{Y_{G}^{H}}\right. \\
& \left.\times \sum_{\alpha \beta} \frac{1+\Gamma_{\alpha}}{Y_{\alpha}} M_{\alpha \beta}^{-1}\left\langle\beta \mid G_{0} P\right\rangle\langle G P \mid \alpha\rangle\right) A_{0}^{-},
\end{aligned}
$$

where the matrix elements $M_{\alpha \beta}$ are defined as

$$
M_{\alpha \beta}=\left(1-\Gamma_{\alpha}\right) \delta_{\alpha \beta}-\left(1+\Gamma_{\beta}\right) \chi_{\alpha \beta},
$$

and the interaction sums are given by

$$
\chi_{\alpha \beta}=\sum_{G} H_{G G}^{\alpha \beta} \frac{Y_{G P}^{d}}{Y_{\alpha}} \frac{Y_{G P}^{0} \cos \left(p_{G} \ell\right)-i Y_{G P}^{d} \sin \left(p_{G} \ell\right)}{-i Y_{G P}^{0} \sin \left(p_{G} \ell\right)+Y_{G P}^{d} \cos \left(p_{G} \ell\right)},
$$

with

$$
H_{G G}^{\alpha \beta}=\langle\alpha \mid G P\rangle\langle G P \mid \beta\rangle .
$$

Figure 8 shows the absorbance spectra for a square lattice containing four cavities per unit cell. All the four cavities have the same inner and outer radii, given by $r_{i} / a=0.1$ and $r_{e} / a=$ 0.2 , respectively, but different depths, being $h_{1} / a=0.35$, $h_{2} / a=0.4, h_{3} / a=0.45$, and $h_{4} / a=0.5$. The dielectric slab with thickness $\ell / a=0.1$ has a permittivity $\varepsilon=2+i 0.1$. This 


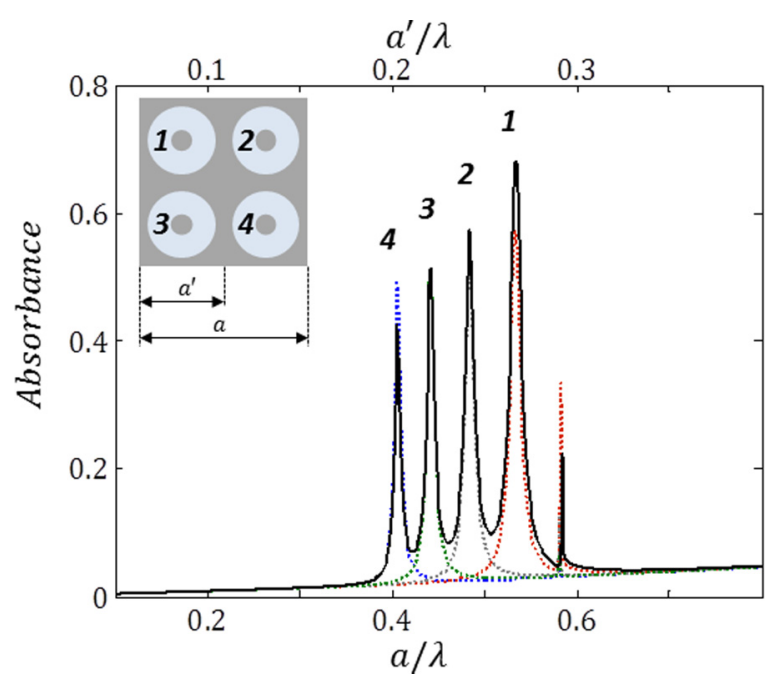

FIG. 8. (Color online) Absorbance of dielectric slab with thickness $\ell / a=0.1$ and permittivity $\varepsilon_{d}=2+i 0.1$ on top of a metallic metasurface containing four annular cavities in the units cell (see the inset). The four cavities have the same cylindrical section $\left(r_{i 1,2,3,4} / a=0.1, r_{e 1,2,3,4} / d=0.2\right)$, but their lengths are different: $h_{1} / a=0.35, h_{2} / a=0.4, h_{3} / a=0.45$, and $h_{4} / a=0.5$. Results are obtained for the incident angles $\phi=0$ and $\theta_{0}=\pi / 4$.

result is obtained for the incident angle is $\theta_{0}=\pi / 4$. It is clear that there is no coupling between the cavity resonances, since the response of the complete system can be understood as the superposition of the responses of four different cells, each one having one of the holes and respective depth $h_{1}, h_{2}, h_{3}$, or $h_{4}$, which has been depicted in the figure by dotted lines. This lack of interaction can be confirmed by the fact that the interaction matrix $\chi_{\alpha \beta}$ has only significant values in the diagonal elements when $\alpha=\beta$. The individual resonances of each cavity have very low coupling to neighboring cells and, therefore, these supercells can be employed for designing multifrequency absorbing layers.

\section{B. Guided modes in slab}

The excitation of guided modes of the dielectric layer can also produce absorptive peaks. These modes propagate along the lateral dimension of the slab and, consequently, their optical paths can be much larger than that corresponding to the vertical dimension. Remember that the excitation of these modes from the far field is not possible since the parallel wave number required for this excitation cannot be provided by a free propagating wave. The role of the array of resonators is precisely to provide this additional wave number by the excitation of a set of diffracted waves.

Figure 9(a) shows the absorbance spectra for three values of the permittivity: $\varepsilon_{d}=2(1+i 0.05)$ (continuous line), $\varepsilon_{d}=$ $2(1+i 0.005)$ (dashed line), and $\varepsilon_{d}=2(1+0.15 i)$ (dotted line). Results are obtained for the incident direction defined by the angles $\theta=\pi / 4$ and $\phi=0$, and the parameters of the structure are reported in the caption. The absorptive peaks denoted by $G_{1}, G_{2}$, and $G_{3}$ are caused by the excitation of hybrid modes that are guided along the dielectric slab. They
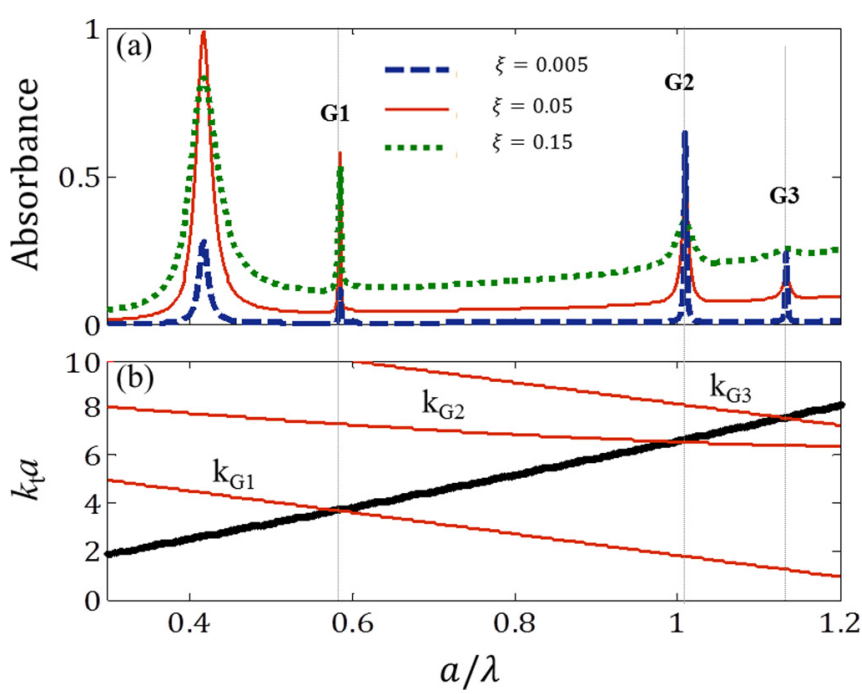

FIG. 9. (Color online) (a) Absorbance with a dielectric slab of thickness $\ell / a=0.1$ and permittivity $\varepsilon_{d}=2(1+i \xi)$. Absorbance has been calculated for three values of $\xi$. (b) Dispersion diagram of the guided modes inside the slab. The annular cavities on the metallic metasurface have dimensions $r_{i} / a=0.1, r_{e} / a=0.4$, and $h / a=0.4$. Results are calculated for the incidence angles $\theta_{0}=\pi / 4$ and $\phi=0$.

are excited thanks to the transverse component $k_{G_{i}}$ of the diffracted modes, as explained below.

The $P$-polarized guided modes of a dielectric slab with a metallic grating underneath are obtained by setting $C_{\alpha}=0$ in Eq. (21) and inserting the condition obtained for $B_{G P}^{ \pm}$into Eqs. (17) and (18). In addition, by assuming that there is no incident field, the condition for the existence of a guided mode can be written as

$$
-i Y_{G P}^{0} \sin \left(p_{G} \ell\right)+Y_{G P}^{d} \cos \left(p_{G} \ell\right)=0 .
$$
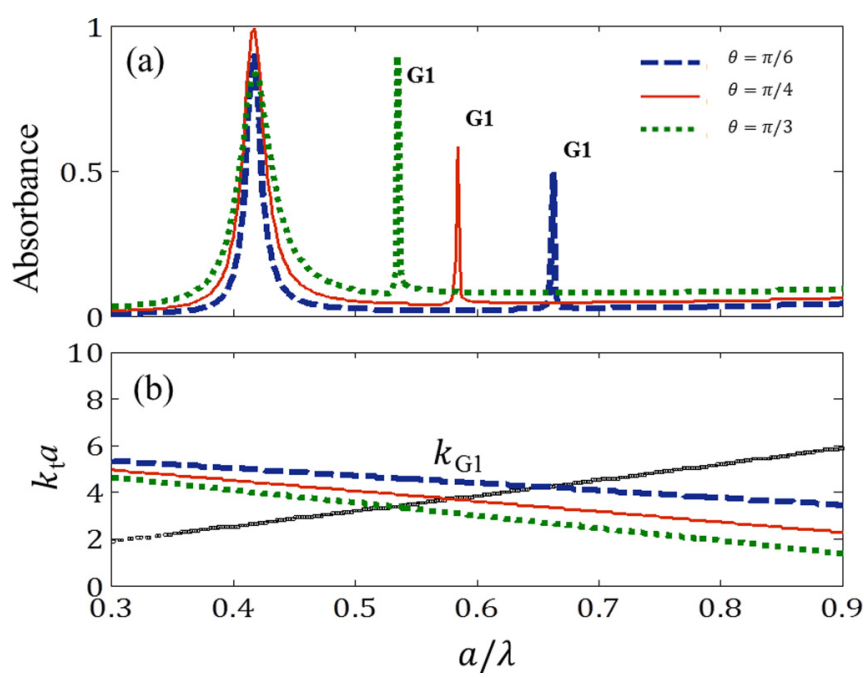

FIG. 10. (Color online) Dependence of the absorbance for the incident angle $\theta$ at fixed $\phi=0$. Results are obtained with a slab with $\ell / a=0.1$ and permittivity $\varepsilon_{d}=2(1+i 0.05)$. (b) Dispersion diagram of the guided modes inside the slab. The annular cavities on the metallic metasurface have dimensions $r_{i} / a=0.1, r_{e} / a=0.4$, and $h / a=0.4$. 
This expression is contained in the denominator of the term $\chi(\omega)$ [see Eq. (36)]. When this condition is fulfilled a peak in the absorbance spectrum will appear, as shown in Fig. 9(b), where the dispersion diagram of the dielectric slab (black line) and the transverse components of the diffracted modes (dotted lines) are depicted. Notice that the crossing points between both of them mark the peak positions. The reciprocal vectors at which the peaks appear are $\mathbf{G}_{1}=(-1,0), \mathbf{G}_{2}=(-1,1)=$ $(1,-1)$, and $\mathbf{G}_{3}=(-2,0)$, using the labeling $\mathbf{G}=\left(m_{1}, m_{2}\right)$ of Eq. (1).

The dependence on the incident direction is depicted in Fig. 10 where it is clearly shown the angle dependence predicted by condition (52). Notice that, on the contrary, no dependence is found for the low-frequency peak, which corresponds to the fundamental cavity mode.

\section{SUMMARY}

In summary, we have developed an analytical model for studying the absorptive properties of structures consisting of a very thin and lossy dielectric slab on top of a metasurface. This model has allowed us to comprehensively analyze the absorbance of $P$-polarized EM waves impinging a lossy dielectric backed with a metasurface consisting of a metallic plate with a periodic arrangement of coaxial or annular cavities. An analytical expression for the absorbance has been obtained under the assumption that only the fundamental mode of cavity array is excited. The results obtained from this expression have been corroborated by full-wave simulations based on finite elements.

It has been shown that a perfect absorption (unity absorbance peak) can be obtained as a consequence of the interaction of the dielectric slab with the excited fundamental mode of the annular cavity. This narrow resonant peak appears at wavelengths one order of magnitude larger than the thickness of the dielectric slab and the conditions for its frequency position and amplitude have been demonstrated. It was found that its frequency position mainly depends on the cavity length and that perfect absorption (unity amplitude) is mainly determined by the absorptive properties of the dielectric slab. Therefore, both properties can be tailored at will by using our analytical expressions.

Also, two additional mechanisms for EM absorption by these structures have been considered. The first mechanism considers the possibility of creating supercells with more than one cavity per unit cell, which is especially useful to design multifrequency absorbers. The other mechanism studied consists of the excitation of guided waves in the dielectric layer, which also produces absorbance peaks but much weaker than that featured at low frequencies.

Hopefully, the results reported here will stimulate experiments confirming the predictions. Although the results reported here are polarization sensitive and for oblique incidence, the present analysis can be applied to other metasurfaces containing different types of resonators and polarizations. For a continuation of this work the goals are the design of metasurfaces producing omnidirectional absorbance by the dielectric film at any polarization and with broadband properties.

\section{ACKNOWLEDGMENTS}

This work was partially supported by the Spanish Ministerio de Economia y Competitividad (MINECO) under contract TEC2010-19751 and by the US Office of Naval Research.

\section{APPENDIX A: OVERLAP INTEGRALS}

The application of boundary conditions by mode-matching requires the calculation of the overlap integrals

$$
\begin{aligned}
\left\langle\boldsymbol{k}_{G} \sigma \mid \alpha\right\rangle & =\iint_{\Omega_{c}}\left\langle\boldsymbol{k}_{G} \sigma \mid \boldsymbol{r}\right\rangle \cdot\langle\boldsymbol{r} \mid \alpha\rangle d \Omega \\
& =\iint_{\Omega_{c}}\left\langle\boldsymbol{r} \mid \boldsymbol{k}_{G} \sigma\right\rangle^{*} \cdot\langle\boldsymbol{r} \mid \alpha\rangle d \Omega,
\end{aligned}
$$

where the surface integral is over the coaxial aperture. The electric field inside the coaxial apertures corresponding to the TEM mode is given by [24]

$$
\langle\boldsymbol{r} \mid \alpha\rangle=\nabla_{t} \psi_{\mathrm{TEM}},
$$

where $\psi_{\text {TEM }}$ is a solution of Laplace's equation

$$
\nabla^{2} \psi_{\mathrm{TEM}}=0
$$

The calculation of the overlap integrals (A1) is better done if the $S$ and $P$ modes above the plate are expressed as

$$
\left\langle\boldsymbol{r} \mid \boldsymbol{k}_{G} P\right\rangle=\frac{1}{\sqrt{\Omega}} \frac{-i}{k_{G}} \nabla_{t} e^{i \boldsymbol{k}_{G} \cdot \boldsymbol{r}},
$$

and

$$
\left\langle\boldsymbol{s} \mid \boldsymbol{k}_{G} S\right\rangle=\frac{1}{\sqrt{\Omega}} \frac{-i}{k_{G}} \hat{z} \times \nabla_{t} e^{i \boldsymbol{k}_{G} \cdot \boldsymbol{r}} .
$$

Then, for $P$-polarized waves, the overlap integral is

$$
\left\langle\boldsymbol{k}_{G} P \mid \beta\right\rangle=\frac{-i}{k_{G} \sqrt{\Omega}} \iint_{\Omega_{c}} \nabla_{t} e^{i \boldsymbol{k}_{G} \cdot \boldsymbol{r}} \cdot \nabla_{t} \psi_{\mathrm{TEM}} d \Omega,
$$

which, by using Green's first identity, can be expressed as

$$
\begin{aligned}
\left\langle\boldsymbol{k}_{G} P \mid \beta\right\rangle= & \frac{i}{k_{G} \sqrt{\Omega}}\left(\iint_{\Omega_{c}} e^{i \boldsymbol{k}_{G} \cdot \boldsymbol{r}} \nabla_{t}^{2} \psi_{\mathrm{TEM}} d \Omega\right. \\
& \left.-\int_{\partial \Omega_{c}} e^{i \boldsymbol{k}_{G} \cdot \boldsymbol{r}} \hat{\boldsymbol{n}} \cdot \nabla_{t} \psi_{\mathrm{TEM}} d l\right) .
\end{aligned}
$$

The first term of the above integral vanishes because of $\nabla_{t}^{2} \psi_{\text {TEM }}=0$, and the second term can be easily obtained; therefore, the expression for the overlap with $P$-polarized waves is given by

$$
\left\langle\boldsymbol{k}_{G} P \mid \alpha\right\rangle=-i \frac{\sqrt{2 \pi} b}{\sqrt{\Omega}} \frac{e^{i \boldsymbol{k}_{G} \cdot \boldsymbol{R}_{\alpha}}}{\sqrt{\ln (b / a)}} \frac{J_{0}\left(k_{G} b\right)-J_{0}\left(k_{G} a\right)}{k_{G} b} .
$$

Similarly, for $S$-polarized waves, the overlap integral is written as

$$
\left\langle\boldsymbol{k}_{G} S \mid \alpha\right\rangle=\frac{-i}{k_{G} \sqrt{\Omega}} \iint_{\Omega_{c}}\left(\hat{z} \times \nabla_{t} e^{i \boldsymbol{k}_{G} \cdot \boldsymbol{r}}\right) \cdot \nabla_{t} \psi_{\mathrm{TEM}} d \Omega .
$$

Again, by applying Green's first identity and using $\nabla^{2} \psi_{\mathrm{TEM}}=$ 0 , we get

$$
\left\langle\boldsymbol{k}_{G} S \mid \alpha\right\rangle=\frac{-i}{k_{G} \sqrt{\Omega}} \int_{\partial \Omega} \psi_{\mathrm{TEM}} \hat{\boldsymbol{r}} \cdot\left(\hat{z} \times \nabla_{t} e^{i \boldsymbol{k}_{G} \cdot \boldsymbol{r}}\right) d l .
$$


However, the above integral cancels, since boundary conditions for the TEM mode requires that $\psi_{\mathrm{TEM}}=0$ on the aperture's boundary, where the above integral is performed. Thus, $\left\langle\boldsymbol{k}_{G} S \mid \alpha\right\rangle=0$.

\section{APPENDIX B: REAL AND IMAGINARY PARTS OF $\chi$}

The properties derived for the low-frequency absorption peak analyzed in Appendix A requires the identification of the real and imaginary parts of the lattice-sum term $\chi$ defined by Eq. (27). This identification is not easy to do in general, due to the complex form of this term in addition to the presence of the complex dielectric constant of the slab. However, for the current situation, some approximations can be made. The lattice-sum term can be decomposed into the term for $\boldsymbol{G}=0$ and the sum for $\boldsymbol{G} \neq 0$ :

$$
\chi=\chi_{0}+\sum_{G \neq 0} \chi_{G}
$$

It is shown in the text that, under the thin-film approximation, the term $\chi_{0}$ is a real quantity; thus, we only need to identify the real and imaginary parts of the term $\chi_{G}$. Given that the main complexity appears because of the presence of the complex dielectric constant of the slab, we will extract it from the expressions by making a Taylor expansion of $\chi_{G}$ around the loss factor $\xi$, which is assumed to be a small quantity, thus

$$
\chi_{G}(\xi) \approx \chi_{G}(\xi=0)+\xi \partial_{\xi} \chi_{G}(\xi=0) .
$$

The first term of the expansion is found to be purely imaginary, since it is given by

$$
\chi_{G}(\xi=0)=H_{G G}^{2} \frac{Y_{G P}^{d}}{Y_{\alpha}} \frac{Y_{G P}^{0} \cos \left(p_{G} \ell\right)-i Y_{G P}^{d} \sin \left(p_{G} \ell\right)}{-i Y_{G P}^{0} \sin \left(p_{G} \ell\right)+Y_{G P}^{d} \cos \left(p_{G} \ell\right)},
$$

and, below the diffraction limit, the propagation constants $p_{G}$ when $\xi=0$ are purely imaginary, and so are the impedance terms $Y_{G P}^{0}$ and $Y_{G P}^{d}$. Then, it is easy to see that, in the above equation, the term $H_{G G}^{2}$ is real, while the fraction $Y_{G P}^{d} / Y_{\alpha}$ is purely imaginary. The last term in the expression is real because $\cos \left(p_{G} \ell\right)$ and $i \sin \left(p_{G} l\right)$ are also real and the $i$ factor in all the impedances cancel each other.

The second term in the expression has a more complex form, since it is the derivative $\chi_{G}$ with respect to $\xi$ for $\xi=0$ and, after some tedious but straightforward analysis, is given by

$$
\chi_{G}^{\prime}(\xi=0)=\sum_{G \neq 0} H_{G G}^{2} \frac{Y_{G}^{d}}{Y_{\alpha}} \frac{2 \ell k_{d}^{2} p_{G}\left(Y_{G}^{d^{2}}-Y_{G}^{0^{2}}\right)+\left(k_{G}^{2}-p_{G}^{2}\right)\left[2 i Y_{G}^{d} Y_{G}^{0}\left(1-\cos 2 p_{G} \ell\right)-\left(Y_{G}^{d^{2}}+Y_{G}^{0^{2}}\right) \sin 2 p_{G} \ell\right]}{4 p_{G}^{2}\left(Y_{G}^{d} \cos p_{G} \ell-i Y_{G}^{0} \sin p_{G} \ell\right)^{2}},
$$

which is found to be real. Effectively, the term $H_{G G}^{2}$ is real, while the following fraction is purely imaginary. Exploring the numerator of the second term, it is easy to see that all the terms are purely imaginary: the first one is linear in $p_{G}$, the second one contains the factor $2 i$, and the third one is proportional to $\sin p_{G} \ell$. This term multiplied by the first fraction gives us a real number. Finally, since the denominator is real, it is found that the full expression is real.

[1] L. Rayleigh, Philos. Mag. 39, 225 (1920).

[2] M. B. Sobnack, W. C. Tan, N. P. Wanstall, T. W. Preist, and J. R. Samples, Phys. Rev. Lett. 80, 5667 (1998).

[3] T. López-Rios, D. Mendoza, F. J. García-Vidal, J. SánchezDehesa, and B. Pannetier, Phys. Rev. Lett. 81, 665 (1998).

[4] J. A. Porto, F. J. García-Vidal, and J. B. Pendry, Phys. Rev. Lett. 83, 2845 (1999).

[5] R. W. Wood, Proc. R. Soc. London A 18, 269 (1902).

[6] T. Ebbesen, J. Lezec, H. Ghaemi, T. Thio, and P. Wolff, Nature (London) 391, 667 (1998).

[7] F. I. Baida and D. Van Labeke, Phys. Rev. B 67, 155314 (2003).

[8] M. J. Lockyear, A. P. Hibbins, J. R. Sambles, and C. R. Lawrence, Phys. Rev. Lett. 94, 193902 (2005).

[9] V. Lomakin, S. Li, and E. Michielssen, Microwave Opt. Technol. Lett. 49, 1554 (2007).

[10] A. Roberts, Opt. Express 18, 2528 (2010).

[11] F. Baida, A. Belkhir, O. Arar, E. Barakat, J. Dahdah, C. Chemrouk, D. V. Labeke, C. Diebold, N. Perry, and M.-P. Bernal, Micron 41, 742 (2010).

[12] J. S. White, G. Veronis, Z. Yu, E. S. Barnard, A. Chandran, S. Fan, and M. L. Brongersma, Opt. Lett. 34, 686 (2009).
[13] E. Lansey, I. R. Hooper, J. N. Gollub, A. P. Hibbins, and D. T. Crouse, Opt. Express 20, 24226 (2012).

[14] N. Engheta and R. W. Ziolkowski, Metamaterials: Physics and Engineering Explorations (Wiley, Hoboken, New Jersey, 2006).

[15] R. Marqués, F. Martín, and M. Sorolla, Metamaterials with Negative Parameters: Theory, Design and Microwave Applications, Vol. 183 (John Wiley \& Sons, New York, 2008).

[16] N. I. Landy, S. Sajuyigbe, J. J. Mock, D. R. Smith, and W. J. Padilla, Phys. Rev. Lett. 100, 207402 (2008).

[17] H. Tao, N. I. Landy, C. M. Bingham, X. Zhang, R. D. Averitt, and W. J. Padilla, Opt. Express 16, 7181 (2008).

[18] Y. Cheng, Y. Nie, and R. Gong, Opt. and Laser Technol. 48, 415 (2013).

[19] F. Baida and D. Van Labeke, Opt. Commun. 209, 17 (2002).

[20] M. I. Haftel, C. Schlockermann, and G. Blumberg, Phys. Rev. B 74, 235405 (2006).

[21] N. W. Ashcroft and N. D. Mermin, Solid State Physics (HoltSaunders Int. Edition, 1976).

[22] R. E. Collin, Field Theory of Guide Waves, 2nd ed. (IEEE, New York, 1991).

[23] H. F. S. S. (HFSS), Ansys, v.14 (2014).

[24] J. Jackson, Classical Electrodynamics (John Wiley \& Sons, New York, 1998). 